\title{
High-Volume Return Premium And Volume-Liquidity Premium
}

\author{
Megan Y. Sun, Ph.D., University of Wisconsin - River Falls, USA
}

\begin{abstract}
This study investigates the interaction of volume-liquidity premium and high-volume return premium by simultaneously considering two factors that significantly impact future stock returns: trading volume norms and trading volume extremes. The study finds that high-volume return premium does exist. However, the high-volume return premium behaves differently for liquid and illiquid stocks. The high-volume return premium disappears very quickly for illiquid stocks, while it persists much longer for highly liquid stocks. The study also shows evidence that supports volume-liquidity premium. But the volume-liquidity premium behaves differently after stocks experience an extremely high/low volume shock. The volume-liquidity premium only exists for small size stocks after an extremely low volume shock, but this volume-liquidity premium totally disappears for stocks experiencing an extremely high volume shock.
\end{abstract}

Keywords: Trading Volume; High-Volume Return Premium; Market Efficiency

\section{INTRODUCTION}

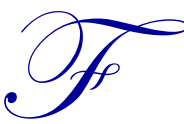

inance scholars have long recognized that past trading volume provides valuable information about the future behavior of stock prices. However, the exact relationship between past trading volume and the direction of future stock returns has yet to be fully investigated. Prior research has focused either on the predictive power of trading volume norms ${ }^{1}$ during certain time intervals (see Datar, Naik and Radcliffe, 1998, Chordia, Subrahmanyam and Anshuman, 2001, and Lee and Swaminathan, 2000) or on the effect which a particular day's trading volume extremes ${ }^{2}$ will have on future stock returns (see Gervais, Kaniel, and Mingelgrin, 2001, Garfinkel and Sokobin, 2006, Lerman, Livnat, and Mendelhall, 2011, Kanile, Ozoguz, and Starks, 2012).

Stocks with high trading volume norms have higher average turnover ratios, and are thus regarded as more liquid. According to a common explanation of stock behavior based on liquidity, stocks with low volume norms earn higher future returns because less liquid stocks require a higher volume-liquidity premium, while stocks with high volume norms earn lower future returns. The existence of this "volume-liquidity premium" is confirmed by Datar, Naik and Radcliffe (1998) and Chordia, Subrahmanyam and Anshuman (2001). These findings are contradicted, however, by Lee and Swaminathan (2000). The contradictory nature of these results suggests a need for further inquiry.

Prior research about "high-volume return premium" also suggests a need for further investigation. Gervais, Kaniel and Mingelgrin (2001) clearly document the existence of high-volume return premium, in which stocks experiencing extremely high volume over a day or a week tend to experience positive excess return, while stocks experiencing extremely low volume over a day or a week tend to subsequently experience negative excess return. The authors argue that the documented high-volume return premium can be explained by the visibility hypothesis which asserts that volume shocks will attract investor's attention towards a particular stock and thus more analysts and traders will become interested in the stock. As previous literature suggests, the arrival of additional analysts and traders for a given stock should increase the stock's value since their input reduces the estimation risk. Later,

\footnotetext{
1 "Trading volume norms" refers here to the average level of trading volume over a significant period of time.

2 "Trading volume extremes" refers here to Gervais, Kaniel and Mingelgrin (2001)'s definition of the term: unusually high or low trading volume when compared to the normal trading volume.
}

(C) 2013 The Clute Institute http://www.cluteinstitute.com/ 
Garfinkel and Sokobin (2006) also document a positive correlation between abnormal share trading volume in the three-day period around earnings announcement and abnormal returns over the subsequent 60 trading days, so do Lerman, Livnat, and Mendenhall (2011). Kanile, Ozoguz and Starks (2012) also observe similar premiums in about 41 countries including both developed equity markets and emerging equity markets as well.

An examination of prior literature reveals that trading volume norms and extremes have always been studied separately. However, both volume norms and volume extremes can significantly influence future returns, an argument made by the liquidity and visibility hypothesis. On days without extremely high or low trading volume, volume norms dominate the determination of future stock returns, while on days exhibiting volume extremes, these extremes work together with volume norms to influence future returns. Stocks exhibiting extreme volumes differ considerably in volume norms - that is, stocks with extreme volumes can be highly liquid or highly illiquid. The high-volume return premium could have different effects on highly liquid stocks and less liquid ones. Therefore, this study places both factors - volume norms and volume extremes - into a single framework of analysis, and then evaluates the effect of one factor on future stock prices relevant to the other factor.

This study contributes to finance literature in two ways. First, this study clarifies the role of high-volume return premium against volume-liquidity premium and finds that high-volume return premium diminishes quickly for stock with less liquidity, while the high-volume return premium last much longer for more liquid stocks. This correlation is robust regardless of the sizes of the stocks. Therefore, we conclude that the effect of high-volume return premium on future stock returns is affected by the liquidity level of stocks.

Second, this study extends the findings of previous literature. Most prior studies establish that trading volume norms are a proxy for liquidity, and that stocks with low volume norms tend to earn higher future returns than those with high volume norms. This study, however, finds that the volume-liquidity premium only holds true for small capitalization stocks after an extremely low volume shock. The volume-liquidity premium totally disappears for stocks experiencing an extremely high volume shock.

The remainder of the study is organized into four more sections. Section two reviews prior finance literature related to our research. Section three provides the data used in our study. Section four describes the methodology used. Section five presents the empirical results attained in relation to the study's main hypothesis. Section six concludes our study with a summary of the evidence.

\section{PREVIOUS RESEARCH}

Trading volume norms refer to the average level of trading volume over a significant period of time, while trading volume extremes refer to the existence of unusually high or unusually low volumes on a particular day. Finance scholars have not incorporated both phenomena into a single framework; they have consistently kept these factors separate.

In general, liquidity measures the ability of shareholders to sell significant amounts of securities quickly with relatively little price impact. In prior literature, trading volume norms is often used as a proxy for liquidity. If investors prefer liquidity, stocks with low volume norms tend to earn higher future returns than those with high volume norms since they are less liquid. However, empirical evidence regarding this issue is very mixed. Datar, Naik, and Radcliffe (1998), among others, study trading volume norms, showing that low turnover stocks generally earn higher returns than high turnover stocks over the next three to five years - a piece of evidence that has supported using trading volume norms as a proxy for liquidity. A study conducted by Chordia, Subrahmanyam, and Anshuman (2001) examines the issue on the firm level rather than the aggregate market level. They use monthly data from a sample of common stocks listed on the NYSE and AMEX to show that trading volume (as measured by either dollar volume or share turnover) is indeed a proxy for liquidity. Their paper clearly documents that, after controlling for various factors (including size, book-to-market ratio, momentum, and dollar volume or share turnover), a strong negative cross-sectional relationship exists between stock returns and the variability of dollar trading volume, and between stock returns and the variability of share turnover at weekly intervals. Based on all firms listed on the NYSE and AMEX from January 1965 to December 1995, Lee and Swaminathan (2000) contradict the above findings in intervals of 3 -months to 5 years. They establish a correlation between the volume 
of any given stock and its average returns in each of the five years prior to portfolio formation. Their findings also indicate that a similar correlation exists between high past trading volumes, glamour characteristics, and more negative earnings surprises over the following eight quarters. On the other hand, low past trading volumes indicate value characteristics and more positive earnings surprises in the future. They conclude that average daily volume is not a proxy for liquidity.

In summary, prior research has yielded contradictory results when examining whether trading volume norms is a proxy for liquidity, where Datar, Naik, and Radcliffe (1998) and Chordia, Subrahmanyam, and Anshuman (2001) support the liquidity hypothesis, Lee and Swaminathan (2000) reach a contradictory conclusion. Further study on the issue is therefore warranted.

As trading volume norms are the subject of a heated debate in finance literature, the status of extreme trading volumes is even hazier, as little research has investigated the effects of volume extremes on subsequent returns. The first empirical work to demonstrate the existence of a high-volume return premium is that of Gervais, Kaniel, and Mingelgrin (2001). Based on stocks traded on the NYSE from 1963 to 1996, the study finds that stocks experiencing extremely high trading volumes over a day- or week-long period tend to appreciate over the course of the following months while those experiencing low extremes will subsequently depreciate. Shocks in a stock's trading volume thus clearly influence subsequent demand and price. This finding indicates the existence of a highvolume return premium and validates the visibility hypothesis. However, the conclusion of Gervais, Kaniel, and Mingelgrin (2001) - that trading volume is not a proxy for liquidity - can be questioned, as they confuse volume extremes with volume norms and they only focus on volume extremes. Later, Garfinkel and Sokobin (2006) document a positive correlation between abnormal share trading volume in the three-day period around earnings announcement and abnormal returns over the subsequent 60 trading days. They interpret the high-volume return premium as compensation for differential risk levels across stocks. They believe higher volume implies greater divergence in investor opinion about firm value and higher stock riskiness. Lerman, Livnat, and Mendenhall (2011) also find a positive relationship between earnings announcement period trading volume and the subsequent returns. Most recently, Kanile, Ozoguz, and Starks (2012) extend the literature on high-volume return premium across border to 41 countries and find that the high-volume return premium, as documented by Gervais, Kaniel, and Mingelgrin (2001) for the United States, is persistent in almost all developed equity markets and in emerging equity markets as well. However, as in Gervais, Kaniel, and Mingelgrin (2001), Kanile, Ozoguz, and Starks (2012) only concentrate on volume extremes and fail to distinguish volume extremes from volume norms.

After a close review of the above finance literature, one can easily infer that the influences of volume norms and volume extremes on future returns are totally different. Volume norms affect future returns through liquidity: stocks with less volume norms tend to be less liquid and are expected to have higher future returns if liquidity matters. Volume extremes affect future returns through visibility: stocks experiencing high volume extremes increase their visibility and are expected to have higher future returns. As volume-liquidity premium and the visibility hypothesis suggest, future returns are influenced by both volume norms and volume extremes. Since both volume norms and volume extremes affect future returns, it would be desirable to estimate the effect of volume norms on future returns by controlling the level of volume extremes and to estimate the effect of volume extremes on future returns by controlling the level of volume norms. However, in prior literature volume norms and volume extremes have always been investigated as independent of each other in their roles in predicting future returns. In order to demonstrate that the high-volume return premium does exist when investigating the effect of volume extremes on future returns, one has to control the level of volume norms. Similarly, in order to show that the volume-liquidity premium exists when investigating the effect of volume norms on future returns, one has to control the level of volume extremes. This study attempts to fill this gap by investigating volume norms and extremes simultaneously.

\section{DATA}

As a starting point, this study gathers data from all of the stocks listed on the NYSE and AMEX during the period July 2, 1962 through December 31, 2010. Daily returns, trading volumes, share turnover and market capitalization data are drawn from the database of the Center for Research in Security Prices (CRSP). NASDAQ firms are not included, as dealer trades are double-counted in the NASDAQ, resulting in trading volume for 
NASDAQ stocks being inflated relative to NYSE and AMEX stocks. ${ }^{3}$ Since we herein rank firms by trading volumes, we would have attained inconsistent results if we mixed firms with different counting procedures. This study also excludes firms that are primes, closed-end funds, mutual firms, real estate investment trusts (REITs), American Depository Receipts (ADRs), or foreign companies. We target the performance of securities identified by CRSP as ordinary common shares (CRSP codes 10 and 11). We then exclude from our sample any stock with missing data on returns, trading volumes, share turnover, or market capitalization during either the portfolio formation period or the holding period.

\section{METHODOLOGY}

As discussed above, this study extends the methodology of Gervais, Kaniel, and Mingelgrin (2001) to investigate the effects of trading volume norms and extremes on subsequent stock returns in a simultaneous analytical framework. Trading volume is measured here in terms of daily turnover ratio, which is defined as the ratio of number of shares traded each day to the number of shares outstanding at the end of the day.

\section{Portfolio formation}

This study splits the time interval from July 2, 1962 to December 31, 2010 into non-intersecting trading intervals of 51 trading days. ${ }^{4}$ The last day of each trading interval is designated as the portfolio formation day. For each eligible stock, the following information is gathered on each formation day: the capitalization of the stock, the volume norms over the formation period, the volume extremes, and the cumulative holding period return 1, 10, 20, 30 , and 50 days after the formation day 5 .

Securities traded in each interval are assigned to three size groups, according to the market capitalization deciles of each firm at the end of this formation day. Following Gervais, Kaniel, and Mingelgrin (2001), firms in market capitalization deciles one to five are assigned to the small capitalization group; firms in deciles six to eight are assigned to the medium group, with firms in deciles nine and ten designated to the large group. ${ }^{6}$ Analysis is then performed separately on each of the three size groups.

This study then ranks all eligible stocks in each size group, based on past trading volume norms over the previous 50 days ending in the formation day. Stocks are then assigned to one of ten portfolios, based on the trading volume norms over the formation period. The study focuses on the bottom decile, stocks with lowest volume norms (N1), and on the top decile, those with highest norms (N10).

The stocks in each resulting size-norm portfolio are then grouped into three categories in accordance with the stock's extreme volume classification on the formation day: extremely low volume portfolios, non-extreme volume portfolios, and extremely high volume portfolios. Extreme volumes are classified as follows. For the 51day formation period used in this paper, if the trading volume on the formation day is among the top 5 out of the 51 daily trading volumes for the formation period, this stock is classified as being of extremely high volume (X3). If the trading volume on the formation day is among the bottom 5 out of the 51 daily trading volumes for the formation period, this stock is classified as being of extremely low volume(X1). Otherwise, it is classified as a non-extreme volume stock(X2).

\footnotetext{
${ }^{3}$ According to CRSP manual, “...[t]here are no volumes available on Nasdaq prior to November 1, 1982. Until June 15, 1992, Nasdaq reported volumes differently on the Nasdaq National Market and Nasdaq Small-Cap Market. On the National Market, the volume of each transaction was reported by one part involved in the transaction. On the Small-Cap Market, all market makers of a security made two volume reports at the end of the market day, specifically, the total number of shares they bought and the total number of shares they sold. The Nasdaq system summed the greater figure (whether buy or sell) from the market reports to create daily volume figures. NYSE/AMEX volumes are the sum of volumes on both exchanges where that security traded that day."

${ }^{4}$ A similar procedure is used in Gervais, Kaniel, and Mingelgrin (2001). Gervais, Kaniel, and Mingelgrin (2001), however, use 50-day intervals, thus causing the same day of the week to appear frequently - a pattern broken only by holidays.

${ }^{5}$ In order to avoid issues arising from overlapping data, the holding period is restricted to being shorter than the formation period.

${ }^{6}$ Firms in the first market decile are not excluded in this paper as they have been elsewhere. These firms potentially provide valuable information and ignoring them in our study could produce biased results.
} 
This study thus focuses on stocks with low volume extremes (X1) and those with high volume extremes (X3) for each size group. For each of these groups, the intersections resulting from these two ranking systems produce 30 norms-extremes portfolios, of which four are of particular interest for our research: N1X1 (low volume norms and low volume extremes), N10X1 (high volume norms and low volume extremes), N1X3 (low volume norms and high volume extremes), and N10X3 (high volume norms and high volume extremes).

\begin{tabular}{|l|l|l|l|}
\hline \multirow{2}{*}{ Combinations for each size group } & Volume Extremes \\
\cline { 3 - 4 } & Low & High \\
\hline \multirow{2}{*}{ Volume Norms } & Low & N1X1 & N1X3 \\
\cline { 2 - 4 } & High & N10X1 & N10X3 \\
\hline
\end{tabular}

(See Appendix A for how to form portfolios based on size, volume norms, and volume extremes)

\section{Calculating average cumulative returns}

The resulting portfolios are held without rebalancing for the subsequent $\mathrm{K}$ holding days (where $\mathrm{K}$ can be 1 , 10, 20, 30, and 50). In calculating the average cumulative returns of these portfolios, we follow Lyon, Barber, and Tsai (1999) and Gervais, Kaniel, and Mingelgrin (2001) to construct a reference return portfolio. Reference return portfolios are constructed by putting equal weights on each stock, adjusting the weight given to each trading interval according to the number of stocks in that interval. These portfolios are held without rebalancing over the entire holding period of $\mathrm{K}$ days, after which the net average cumulative returns of the resulting portfolios are estimated and compared ${ }^{7}$.

The cumulative holding period return for the long (short) position in each reference portfolio is tracked from the first trading day after the formation day throughout the period, using daily returns data and the equation

$\begin{aligned} L R^{b h}= & \frac{\sum_{p=1}^{P} \sum_{i=1}^{n_{s}}\left\{\left[\prod_{t=s}^{s+k}\left(1+R_{i p t}\right)-1\right]-R_{p}\right\}}{N} \\ S R^{b h}= & \frac{\sum_{p=1}^{P} \sum_{i=1}^{n_{s}}\left\{R_{p}-\left[\prod_{t=s}^{s+k}\left(1+R_{i p t}\right)-1\right]\right\}}{N}\end{aligned}$

where $R_{p}=\frac{\sum_{i=1}^{n_{s}}\left\{\left[\prod_{t=s}^{s+k}\left(1+R_{i p t}\right)-1\right]\right\}}{N_{t}}$ is the return of reference portfolio at interval $p . L R^{b h}$ is the cumulative holding period return for the long position and $S R^{b h}$ is the cumulative holding period return for the short position, $R_{i p t}$ is the daily return of security $i$ at time $t$ from interval $p . n_{s}$ is the number of securities traded in interval $s$, the first trading day of each holding period; $k$ is the length of the holding period in days; $P$ is the number of

\footnotetext{
${ }^{7}$ The reference return portfolio strategy is used to avoid the so-called "bad model" problem. Fama (1998b) claims that even under the best scenarios, and while disregarding methodology, all tests for statistically significant extreme returns are contaminated by a "bad model" problem in long-term stock performance studies. He further argues that no asset-pricing model completely describes the systematic average return patterns for any sample period, and that all such models therefore suffer from some form of bias. For short-term studies the so-called "bad model" problem is no issue, as the expected return over a few days is nearly zero in all cases. However, when the time horizon increases, expected returns grow faster than the volatility of these returns, and it is thus easy for studies involving long-term common stock returns to produce false indications of extreme returns. Lyon, Barber, and Tsai (1999) propose several methods for detecting extreme stock returns over long periods and this study follows their buy-and-hold extreme returns (BHAR) approach to calculate average cumulative returns.
} 
holding intervals; $N$ is the total number of securities in the reference portfolio; and the superscript $b h$ represents the buy-and-hold strategy, in which each securities investment is considered passive for the holding period and equally weighted, without balancing.

The average net return of the above portfolios is calculated using the equation:

$$
\overline{N R}^{b h}=\frac{\sum_{p=1}^{P} \sum_{i=1}^{n_{p}}\left(\left\{\left[\prod_{t=s}^{s+\tau}\left(1+R_{i p t}\right)-1\right]-R_{p}\right\}_{\text {Long }}-\left\{R_{p}-\left[\prod_{t=s}^{s+\tau}\left(1+R_{i p t}\right)-1\right]\right\}_{s h o r t}\right)}{N}
$$

Use of the reference return portfolio approach dictates that the weight for each trading interval is adjusted relative to the number of securities in each portfolio. Within each trading interval, however, as discussed above, a reference return portfolio is constructed by equally weighting each of the securities, which are subsequently held without rebalancing. In this way, the average return of the long and short positions may be used separately to test whether the reference return portfolio strategy may generate extreme return.

\section{Expected results}

If trading volume extremes induce a momentum effect in stock prices, stocks with high volume extremes will have higher future returns. If trading volume norms do indeed provide a proxy for liquidity, stocks with low norms will have higher future returns since they are less liquid. Consequently, the following results are expected for the reference return portfolio. First, we expect that significant positive returns will apply to portfolios N1X3-N1X1 and N10X3-N10X1 if high-volume return premium exists. By construction, N1X3 and N1X1 both have low volume norms, which mean they are both less liquid. We can then expect the volume-liquidity premium to be same for N1X3 and N1X1. Since N1X3 is subject to high volume extremes and N1X1 is subject to low volume extremes, the high-volume return premium means we can expect N1X3 to have a higher future return than N1X1. We will observe significant positive returns for portfolio N1X3-N1X1. Similar arguments can be applied to N10X3-N10X1.

Second, the reference return portfolio N1X1-N10X1 and N1X3-N10X3 will show significant positive returns. By construction, N1X1 and N10X1 both have low volume extremes. The effect of volume extremes on future returns should be the same for both portfolios. Since N1X1 is less liquid than N10X1, N1X1is expected to have a higher return than N10X1. That is why portfolio N1X1-N10X1 is expected to have a significant positive return. Similar arguments may apply to N1X3 and N10X3. The following table summarizes the expected results.

\begin{tabular}{|l|l|l|}
\hline & Expected Return & Reason \\
\hline N1X3-N1X1 & Positive & High-volume return premium \\
N10X3-N10X1 & Positive & High-volume return premium \\
N1X1-N10X1 & Positive & Volume-liquidity premium \\
N1X3-N10X3 & Positive & Volume-liquidity premium \\
\hline
\end{tabular}

\section{EMPIRICAL RESULTS}

In order to compare the results from this study with those from Gervais, Kaniel, and Mingelgrin (2001), we first present table 1, which shows the average cumulative returns when grouped only on size and volume extremes. The results in table 1 are very similar to those of Gervais, Kaniel, and Mingelgrin (2001). With a 51-day formation periods, the average net returns of portfolio X3-X1 — long in stocks with high volume extremes and short in stocks with low volume extremes - are clearly positively significant for the overall sample and for all three size groups, at horizons of 1, 10,20,30 and 50 trading days. The results in table 1 clearly indicate that stocks with extremely high volumes generate positively higher returns than stocks with extremely low volumes, at least shortly after the portfolio formation, findings consistent with Gervais, Kaniel, and Mingelgrin (2001). 
Table 1

Average cumulative returns of the zero investment portfolio and reference return portfolio for the 51-day interval In each trading interval, stocks are classified according to size and volume extremes. Size groups are based on the firm's market capitalization at the day prior to each formation period. The volume extremes classification is based on whether the stock's trading volume on the formation day is among the top or bottom $10 \%$ of daily volumes throughout the entire formation period. For each size group, two portfolios are of particular interest to this study. X1: stocks with low volume extremes. X3: stocks with high volume extremes. In all cases, average cumulative returns are calculated for the following different holding periods: 1,10 , 20,30, and 50 trading days. The average cumulative returns are displayed in the first line. The numbers in the second line are tstatistics.

\begin{tabular}{|c|c|c|c|c|c|}
\hline formation-holding period & $51-1$ & $51-10$ & $51-20$ & $51-30$ & $51-50$ \\
\hline \multicolumn{6}{|l|}{ OVERALL } \\
\hline \multirow[t]{2}{*}{$\mathrm{X} 3-\mathrm{X} 1$} & $0.0014 * *$ & $0.0035 * *$ & $0.0045^{* *}$ & $0.0052 * *$ & $0.0043^{* *}$ \\
\hline & 14.86 & 13.01 & 11.79 & 11.13 & 7.08 \\
\hline \multicolumn{6}{|l|}{ SMALL SIZE GROUP } \\
\hline \multirow[t]{2}{*}{$\mathrm{X} 3-\mathrm{X} 1$} & $0.0018 * *$ & $0.0047 * *$ & $0.0060 * *$ & $0.0065 * *$ & $0.0053 * *$ \\
\hline & 11.38 & 9.96 & 9.19 & 8.20 & 5.02 \\
\hline \multicolumn{6}{|l|}{ MEDIUM SIZE GROUP } \\
\hline \multirow[t]{2}{*}{$\mathrm{X} 3-\mathrm{X} 1$} & $0.0011 * *$ & $0.0026 * *$ & $0.0028 * * 4.68$ & $0.0040 * *$ & $0.0037 * *$ \\
\hline & 7.34 & 9.98 & & 5.52 & 3.79 \\
\hline \multicolumn{6}{|l|}{ LARGE SIZE GROUP } \\
\hline \multirow[t]{2}{*}{$\mathrm{X} 3-\mathrm{X} 1$} & $0.0007 * *$ & $0.0017 *$ & $0.0026^{*}$ & $0.0032 * *$ & $0.0030 * *$ \\
\hline & 4.90 & 4.14 & 4.32 & 4.38 & 3.16 \\
\hline
\end{tabular}

Table 2 shows when the volume norm is controlled, the average cumulative returns of the reference return portfolios for the overall sample and each of the three size groups over holding periods of $1,10,20,30$, and 50 days, under 51-day formation period. The average net returns of portfolios N1X3-N1X1 and N10X3-N10X1-after controlling for average volume at both low and high norms, respectively - are clearly positively significant for the overall sample. After controlling for average volume at low norms, the average cumulative returns of portfolio N1X3-N1X1 are significant for the overall sample at least for very short holding periods. The average cumulative returns of portfolio N10X3-N10X1 —after controlling for average volume at high norms - are significant for the overall sample for all five holding periods. The significantly positive profits reported here clearly indicate that stocks with extremely high trading volumes generate positively higher returns than stocks with extremely low trading volumes, which supports the high-volume return premium. Our results also indicate that the high-volume return premium behaves differently for liquid and illiquid stocks. To be more specific, the high-volume return premium diminishes very quickly for stocks that have relatively low volume norms, but the high-volume return premium lasts much longer for stocks that have relatively high volume norms. Therefore, the effect of highvolume return premium on future stock returns is affected by the liquidity level of stocks.

Further study of Table 2 shows that the results found from overall sample are robust for each of the three size groups. The average cumulative returns for stocks with high volume norms are positively significant at all three size groups, while stocks with low volume norms don't show any significant return difference in the three size groups. Therefore the high-volume return premium is more evident and long lasting for highly liquid stocks. 
Table 2

Average cumulative returns of the reference return portfolio for the 51-day interval after controlling volume norms

In each trading interval, stocks are classified according to size, volume norms, and volume extremes. Size groups are based on the firm's market capitalization at the day prior to each formation period. Volume norms refer to the average daily trading volume during the formation periods. The volume extremes classification is based on whether the stock's trading volume at the formation day is among the top or bottom $10 \%$ of the daily volumes in the whole formation period. For each size group, four portfolios are of particular interest to this study. N1X1: stocks with low volume norms and low volume extremes. N10X1: stocks with high volume norms and low volume extremes. N1X3: stocks with low volume norms and high volume extremes. N10X3: stocks with high volume norms and high volume extremes. In all cases, average cumulative returns are calculated for the following different holding periods: 1, 10, 20, 30, and 50 trading days. The average cumulative returns are displayed in the first line. The numbers in the second line are t-statistics.

\begin{tabular}{|c|c|c|c|c|c|}
\hline formation-holding period & $51-1$ & $51-10$ & $51-20$ & $51-30$ & $51-50$ \\
\hline \multicolumn{6}{|l|}{ OVERALL } \\
\hline \multirow[t]{2}{*}{ N1X3-N1X1 } & $0.0008 * *$ & $0.0018 * *$ & 0.0007 & 0.0008 & 0.0005 \\
\hline & 3.23 & 2.63 & 0.70 & 0.76 & 0.32 \\
\hline \multirow[t]{2}{*}{ N10X3-N10X1 } & $0.0021 * *$ & $0.0056 * *$ & $0.0083 * *$ & $0.0097 * *$ & $0.0085^{* *}$ \\
\hline & 5.22 & 4.80 & 4.82 & 4.66 & 2.95 \\
\hline \multicolumn{6}{|l|}{ SMALL SIZE GROUP } \\
\hline \multirow[t]{2}{*}{ N1X3-N1X1 } & 0.0009 & 0.0024 & -0.0002 & 0.0002 & -0.0011 \\
\hline & 1.83 & 1.88 & -0.09 & 0.09 & -0.40 \\
\hline \multirow[t]{2}{*}{ N10X3-N10X1 } & $0.0020 * *$ & $0.0053 * *$ & $0.0076 * *$ & $0.0074 * *$ & 0.0060 \\
\hline & 3.51 & 3.34 & 3.32 & 2.69 & 1.65 \\
\hline \multicolumn{6}{|l|}{ MEDIUM SIZE GROUP } \\
\hline \multirow[t]{2}{*}{ N1X3-N1X1 } & $0.0010 * *$ & 0.0010 & 0.0007 & -0.0004 & 0.0001 \\
\hline & 2.75 & 0.95 & 0.50 & -0.23 & 0.05 \\
\hline \multirow[t]{2}{*}{ N10X3-N10X1 } & $0.0019 * *$ & $0.0048 *$ & $0.0066^{*}$ & $0.0091 *$ & 0.0086 \\
\hline & 2.87 & 2.27 & 2.12 & 2.44 & 1.59 \\
\hline \multicolumn{6}{|l|}{ LARGE SIZE GROUP } \\
\hline \multirow[t]{2}{*}{ N1X3-N1X1 } & 0.0003 & 0.0011 & 0.0011 & 0.0033 & 0.0034 \\
\hline & 0.80 & 1.06 & 0.84 & 1.95 & 1.56 \\
\hline \multirow[t]{2}{*}{ N10X3-N10X1 } & $0.0025 * *$ & 0.0057 & $0.0104 *$ & $0.0187 * *$ & $0.0178 *$ \\
\hline & 2.38 & 1.87 & 2.10 & 3.14 & 2.27 \\
\hline
\end{tabular}

Table 3, on the other hand, shows when the volume extreme is controlled, the average cumulative returns of the reference return portfolios for the overall sample and each of the three size groups over holding periods of 1 , 10, 20, 30, and 50 days, under 51-day formation period. The average net returns of N1X1-N10X1 and N1X3N10X3 similarly controlled for volume extremes at low and high levels respectively, enable us to investigate the other main subject in our study, volume-liquidity premium. The average net returns of N1X1-N10X1 are significantly positive for the overall sample and the small size group. We find that when stocks, especially small stocks, experience an extremely low volume shock, those stocks with low volume norms tend to earn higher returns than those with high volume norms, just as the liquidity explanation postulates. However, the opposite is sometimes observable for stocks experiencing an extremely high volume shock. When we control for volume extremes at high levels, the average net returns of N1X3-N10X3 are often negative for overall sample and the three size groups, though not very statistically significant. Therefore for stocks experiencing high volume extremes, the volume norm is no longer an important factor in determining the future returns. In sum, the empirical results form this study support that volume-liquidity premium is more evident for small size stocks after an extremely low volume shock. The volume-liquidity premium disappears after an extremely high volume shock. 
Table 3

Average cumulative returns of the reference return portfolio for the 51-day interval after controlling volume extremes In each trading interval, stocks are classified according to size, volume norms, and volume extremes. Size groups are based on the firm's market capitalization at the day prior to each formation period. Volume norms refer to the average daily trading volume during the formation periods. The volume extremes classification is based on whether the stock's trading volume at the formation day is among the top or bottom $10 \%$ of the daily volumes in the whole formation period. For each size group, four portfolios are of particular interest to this study. N1X1: stocks with low volume norms and low volume extremes. N10X1: stocks with high volume norms and low volume extremes. N1X3: stocks with low volume norms and high volume extremes. N10X3: stocks with high volume norms and high volume extremes. In all cases, average cumulative returns are calculated for the following different holding periods: 1, 10, 20, 30, and 50 trading days. The average cumulative returns are displayed in the first line. The numbers in the second line are t-statistics.

\begin{tabular}{|c|c|c|c|c|c|}
\hline formation-holding period & $51-1$ & $51-10$ & $51-20$ & $51-30$ & $51-50$ \\
\hline \multicolumn{6}{|l|}{ OVERALL } \\
\hline \multirow[t]{2}{*}{ N1X1-N10X1 } & $0.0007 *$ & $0.0027 * *$ & $0.0033 *$ & $0.0041 *$ & 0.0041 \\
\hline & 2.43 & 3.06 & 2.50 & 2.45 & 1.86 \\
\hline \multirow[t]{2}{*}{ N1X3-N10X3 } & -0.0005 & -0.0009 & $-0.0041 * *$ & $-0.0046 * *$ & -0.0036 \\
\hline & -1.52 & -0.90 & -2.88 & -2.68 & -1.58 \\
\hline \multicolumn{6}{|l|}{ SMALL SIZE GROUP } \\
\hline \multirow[t]{2}{*}{ N1X1-N10X1 } & $0.0009 * *$ & $0.0033 *$ & $0.0046^{*}$ & 0.0042 & 0.0055 \\
\hline & 3.12 & 2.39 & 2.24 & 1.65 & 1.65 \\
\hline \multirow[t]{2}{*}{ N1X3-N10X3 } & -0.0006 & -0.0007 & -0.0043 & -0.0043 & -0.0024 \\
\hline & -0.97 & -0.44 & -1.91 & -1.60 & -0.67 \\
\hline \multicolumn{6}{|l|}{ MEDIUM SIZE GROUP } \\
\hline \multirow[t]{2}{*}{ N1X1-N10X1 } & 0.0002 & 0.0018 & 0.0020 & 0.0048 & 0.0043 \\
\hline & 0.51 & 1.15 & 0.89 & 1.74 & 1.11 \\
\hline \multirow[t]{2}{*}{ N1X3-N10X3 } & -0.0005 & -0.0016 & -0.0033 & -0.0042 & -0.0035 \\
\hline & -0.90 & -1.00 & -1.41 & -1.46 & -0.88 \\
\hline \multicolumn{6}{|l|}{ LARGE SIZE GROUP } \\
\hline \multirow[t]{2}{*}{ N1X1-N10X1 } & 0.0004 & 0.0027 & 0.0019 & 0.0025 & 0.0042 \\
\hline & 0.99 & 1.72 & 0.78 & 0.87 & 1.06 \\
\hline \multirow[t]{2}{*}{ N1X3-N10X3 } & -0.0005 & 0.0009 & -0.0020 & -0.0025 & -0.0004 \\
\hline & -0.88 & 0.58 & -0.87 & -0.87 & -0.11 \\
\hline
\end{tabular}

\section{Economic significance}

Berkowitz, Logue and Noser (1988), using a sample of NYSE firms, estimate that the average transaction cost is $0.23 \%$ for brokers and money managers. Elkins/McSherry (1998) use various samples based on actual trades by institutional investors to examine trading costs. Based on all the trades for which they have data, they estimate the transaction cost for trades on the NYSE is $0.29 \%^{8}$. Recently, Berkowitz and Logue (2001) have reported that total transaction costs can be as low as $0.18 \%$. This lower transaction cost is achievable for institutional investors, who also report having soft dollar and commission recapture arrangements in place, which can further lower their transaction costs. Lower transaction cost is definitely the trend in the market as trading activity increases and competition becomes more heated. Given the fact that the transaction cost can be as low as $0.18 \%$, we may conclude with some certainty that even after accounting for transaction cost, the high-volume return premium and the volume-liquidity premium are still economically profitable at least for large institutional investors.

\section{CONCLUSION}

This study has focused on "volume-liquidity premium" and "high-volume return premium" by investigating the interaction between trading volume norms and trading volume extremes. We have placed particular attention on their effects on future stock returns. Prior literature has produced contradictory results regarding the existence of a volume-liquidity premium and documents the existence of high-volume-return premium within all size groups. Our study confirms the existence of a volume-liquidity premium and a high-volume return premium, similar to the conclusions of Datar, Naik and Radcliffe (1998), Chordia, Subrahmanyam and Anshuman

\footnotetext{
${ }^{8}$ Elkins/McSherry (1998) also finds that the transaction cost for trades on the TSE is $0.29 \%$, while the transaction cost for trades
} on the NASDAQ is $0.35 \%$.

(C) 2013 The Clute Institute http://www.cluteinstitute.com/ 
(2001) and Gervais, Kaniel and Mingelgrin (2001) and Kanile, Ozoguz, and Starks (2012). However, this study also shows that the high-volume return premium behaves differently depending on the liquidity level of stocks. The high-volume return premium lasts much longer for stocks with a high liquidity, while disppears very quickly for less liquid stocks. This study also finds that the volume-liquidity premium exist only for small size stocks after experiencing an extremely low volume shock, while the volume-liquidity premium disappears for those stocks experiencing an extremely high volume shock. In summary, the high-volume return premium works best for high liquid stocks, while the volume-liquidity premium works best for small size stocks after experiencing an extremely low volume shock.

The findings in this study thus add much-needed clarity to the market efficiency debate which is ongoing in finance literature. Our findings regarding the relationships between trading volumes - both norms and extremesand future stock returns provide evidence that trading volumes norms and trading volume extremes both can be used to gauge future prices. Portfolio managers should therefore be aware that trading volumes are statistically significant determinants of future market returns. Those who utilize trading volumes in their decision-making processes will be likely to perform better than those who do not.

\section{AUTHOR INFORMATION}

Megan Y. Sun, Ph.D., is the Associate Professor of Finance in the Department of Accounting and Finance at the University of Wisconsin - River Falls, USA. Her research interests include asset pricing, market efficiency, market microstructure, derivatives, and behavioral finance. E-mail: megan.sun@uwrf.edu

\section{REFERENCES}

1. Berkowitz, S., D. E. Logue, and E. A. Noser (1988), "The total cost of transaction on the NYSE," Journal of Finance 43 (No. 1, February), pp. 97-121

2. $\quad$ Berkowitz, S. and D.E. Logue (2001), “Transaction cost,” Journal of Portfolio Management 27 (No. 2, Winter), pp. 65-75

3. Chordia, T. and A. Subrahmanyam(2000), "Trading volume and cross-autocorrelations in stock returns," Journal of Finance 55 (No. 2, April), pp. 913-935.

4. $\quad$ Chordia, T., A. Subrahmanyam, and V. R. Anshuman (2001), "Trading activity and expected stock returns," Journal of Financial Economics 59 (No. 1, January), pp. 3-32.

5. Datar, V., N. Naik, and R. Radcliffe (1998), "Liquidity and asset returns: an alternative test," Journal of Financial Markets 1, (No. 2, August), pp. 203-220.

6. Elkins/McSherry Co. Inc, (1998), "Transaction costs in Canadian and U.S. equity markets: a study of instituitional trading in cross-listed stocks, "report published by the Toronto Stock Exchange.

7. Fama, E. F.(1998), "Market efficiency, long term returns, and behavorial finance," Journal of Financial Economics 49 (No. 3, September), pp. 286-306.

8. Fama, E. F. (1998), "Determine the number of priced state variables in the ICAPM," Journal of Financial and Quantitative Analysis 33 (No. 2, June), pp. 217-231.

9. Garfinkel, J. A. and J. Sokobin (2006), "Volume, Opinion Divergence, and Returns: a Study of PostEarnings Annoucement Drift," Journal of Accounting Research 44 (No. 1. March), pp 85-112

10. Gervais, S., R.n Kaniel, and D. Mingelgrin (2001), “The high volume return premium," Journal of Finance 56 (No. 3, June), pp. 877-919.

11. Kanile, R., A. Ozoguz, and L. Starks (2012), “The High-Volume Return Premium and the Investor Recognition Hypothesis: International Evidence and Determinants," Journal of Financial Economics 103 (No. 2, February), pp. 255-279.

12. Lerman, A., J. Livnat, and R. R. Mendenhall (2011), "The High-Volume Return Premium and PostEarnings Announcement Drift," Working Paper,

13. Lee, C. and B. Swaminathan (2000), "Price momentum and trading volume," Journal of Finance 55 (No. 5 , October), pp. 2017-1069.

14. Lyon, J. D., B. M. Barber, and C. Tsai (1999), "Improved methods for tests of long-run abnormal stock returns," Journal of Finance 54 (No. 1, February), pp. 165-201. 
APPENDIX A: Portfolio formation strategy for 51-day interval based on size, volume norms and volume extremes

On each formation day, all eligible stocks are assigned to three size groups, according to the market capitalization deciles of each firm at the end of the formation day. Firms in market capitalization deciles one to five are assigned to the small capitalization group; firms in deciles six to eight are assigned to the medium group, with firms in deciles nine and ten designated to the large group. This study then ranks all eligible stocks in each size group, based on past trading volume norms over the previous 51 days ending on the formation day. Stocks are then assigned to one of ten portfolios, based on the trading volume norms over the formation period. The study focuses on the bottom decile, i.e., stocks with low volume norms (N1), and on the top decile, i.e., those with high norms (N10). The resulting portfolios are then divided into three categories: extremely low volume portfolios (X1), non-extreme volume portfolios (X2), and extremely high volume portfolios (X3), according to the stock's extreme volume classification on the formation day. By contrast, extreme volumes are classified as follows. On a given formation day, a stock is classified as extremely high volume if its trading volume is among the top 10 percent of daily trading volumes for the formation period, while an extremely low volume stock is among the bottom 10 percent of daily trading volumes for that period. All other stocks are classified as non-extreme volume stocks.

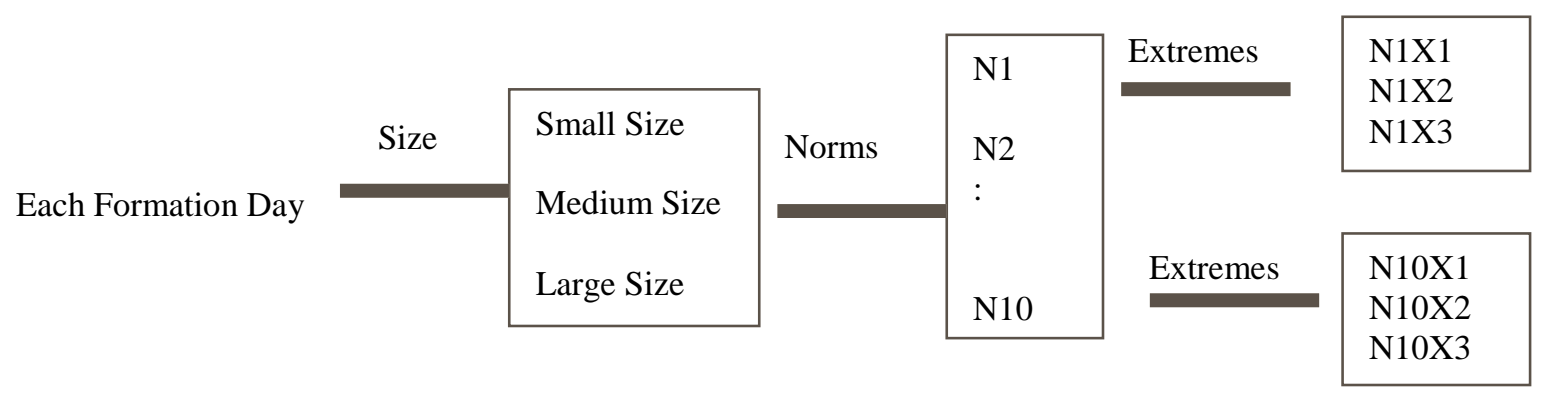




\section{NOTES}

